\title{
Hands-Free Augmented Reality for Vascular Interventions
}

\author{
Alon Grinshpoon \\ Columbia University \\ ag3848@columbia.edu \\ Carmine Elvezio \\ Columbia University \\ ce2236@columbia.edu
}

\author{
Shirin Sadri \\ Columbia University \\ ss5218@columbia.edu \\ Samantha Siu \\ Columbia University \\ ss4313@columbia.edu
}

\author{
Gabrielle J. Loeb \\ Columbia University \\ gj12113@columbia.edu
}

\author{
Steven K. Feiner \\ Columbia University \\ skf1@columbia.edu
}

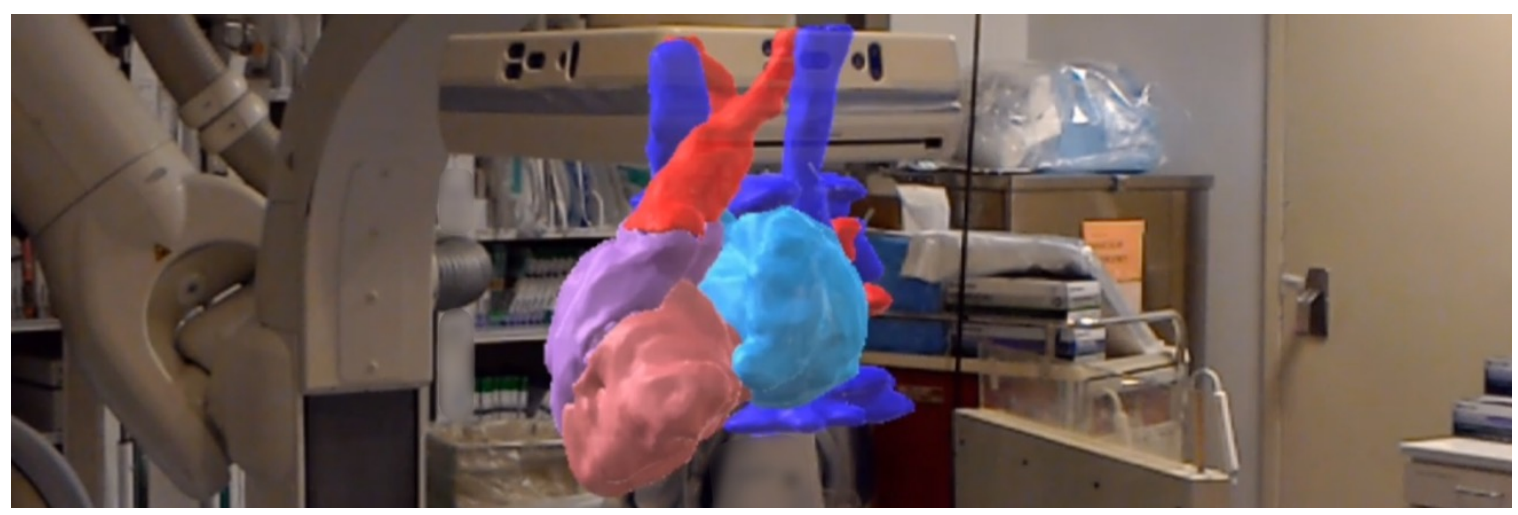

Figure 1: Model of patient heart viewed through HoloLens in procedure room.

\begin{abstract}
During a vascular intervention (a type of minimally invasive surgical procedure), physicians maneuver catheters and wires through a patient's blood vessels to reach a desired location in the body. Since the relevant anatomy is typically not directly visible in these procedures, virtual reality and augmented reality systems have been developed to assist in 3D navigation. Because both of a physician's hands may already be occupied, we developed an augmented reality system supporting hands-free interaction techniques that use voice and head tracking to enable the physician to interact with 3D virtual content on a head-worn display while leaving both hands available intraoperatively. We demonstrate how a virtual 3D anatomical model can be rotated and scaled using small head rotations through first-order (rate) control, and can be rigidly coupled to the head for combined translation and rotation through zero-order control. This enables easy manipulation of a model while it stays close to the center of the physician's field of view.
\end{abstract}

\section{CCS CONCEPTS}

- Human-centered computing $\rightarrow$ Mixed / augmented reality; Graphics input devices; Interaction techniques; User interface

Permission to make digital or hard copies of part or all of this work for personal or classroom use is granted without fee provided that copies are not made or distributed for profit or commercial advantage and that copies bear this notice and the full citation on the first page. Copyrights for third-party components of this work must be honored. For all other uses, contact the owner/author(s).

SIGGRAPH '18 Emerging Technologies, August 12-16, 2018, Vancouver, BC, Canada

(C) 2018 Copyright held by the owner/author(s).

ACM ISBN 978-1-4503-5810-1/18/08.

https://doi.org/10.1145/3214907.3236462 design; Information visualization; - Social and professional topics $\rightarrow$ Medical technologies; • Computing methodologies $\rightarrow$ Graphics input devices;

\section{KEYWORDS}

Hands-free interaction, augmented reality, vascular interventions, head tracking, head-worn display.

\section{ACM Reference Format:}

Alon Grinshpoon, Shirin Sadri, Gabrielle J. Loeb, Carmine Elvezio, Samantha Siu, and Steven K. Feiner. 2018. Hands-Free Augmented Reality for Vascular Interventions. In Proceedings of SIGGRAPH '18 Emerging Technologies. ACM, New York, NY, USA, 2 pages. https://doi.org/10.1145/3214907.3236462

\section{INTRODUCTION}

When performing vascular interventions, physicians frequently need both hands for intraoperative tasks. However, augmented reality (AR) and virtual reality (VR) systems for surgical procedures often rely on hand-based or foot-pedal input, sometimes complemented by head tracking, to enable targeting and selection (e.g., [Hasan and Yu 2017; Jalaliniya et al. 2013; LaValle et al. 2014; Mewes et al. 2017]).

To address the need for hands-free visualization control, without involving the physician's feet, we present the head-tracking and voice-based 3D user interface to an $\mathrm{AR}$ guidance system for vascular interventions that uses Microsoft HoloLens to present 3D models of patient anatomy (Figure 1) [Grinshpoon et al. 2018; Loeb et al. 2018]. Since current AR head-worn displays (HWDs) have a limited field of view (FOV), physicians can potentially lose sight of virtual content during manipulation. To avoid this, we use first-order (rate) 

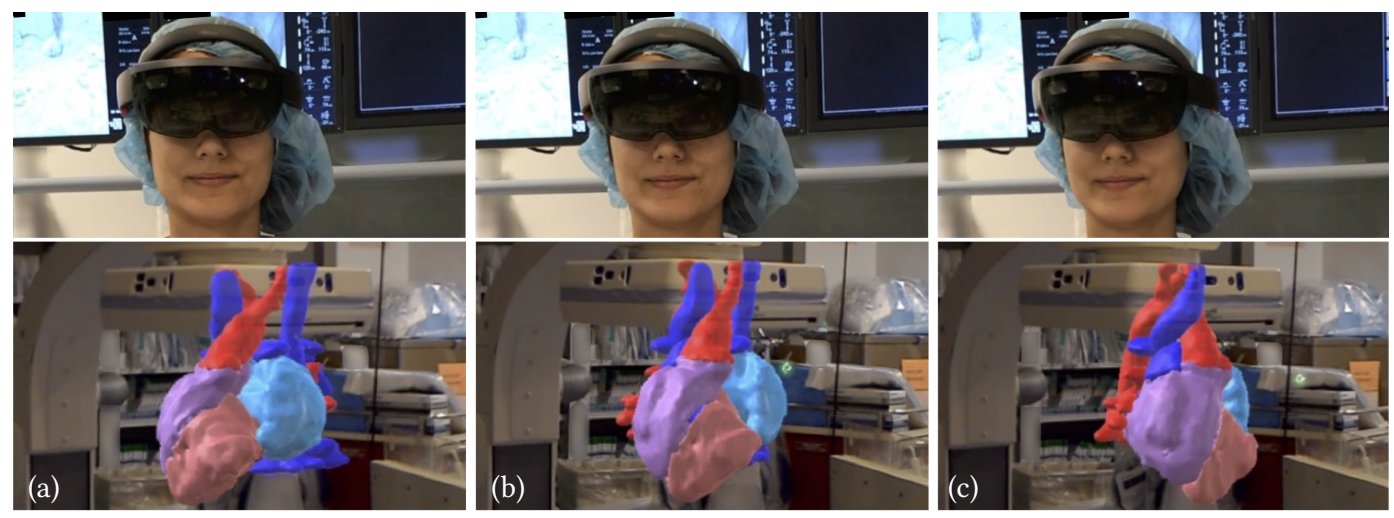

Figure 2: Rotating a model. (a) User selects transformation mode while looking at model. (b) In rotation mode, model rotates in same direction as head (to right, as shown here). (c) The farther away head is oriented from model center, the faster transformation is applied. Only small changes in head orientation are required to control transformation, as shown in top row.

control of model rotation and scaling in response to small head rotations (Figure 2), keeping content near the center of the FOV.

Image-based facial motion and orientation tracking of physicians has been used to control position and orientation of surgical instruments such as laparascopes [Nishikawa et al. 2003] and endoscopic cameras [Reilink et al. 2010; Wachs et al. 2010], intraoperatively using 3DOF zero- and first-order control. However, this may introduce restrictions on a physician's head position and orientation or require physical input through methods such as foot pedals [Reilink et al. 2010] in order to function properly. Because we use a selfcontained, 6DOF-tracked HWD and voice-command control, we avoid these restrictions and enable a physician to move their head and body freely throughout a procedure.

\section{USER INTERFACE}

The user selects a virtual model by positioning and orienting their head such that a cursor aligned with the forward-facing direction of their head intersects with that model. The user then issues a voice command to select a mode, indicated by the cursor icon.

Head translation and rotation control transformation of the model. For rotation (Figure 2) and scaling, the user must look away from the model's center window (a predefined distance from the model center) to apply the transformation, using first-order (rate) control. The user can pause the transformation by looking back towards the center window beyond a predefined cursor window or saying "stop" to exit the transformation completely.

Transformation rate is determined by the magnitude of the vector $\vec{r}$ between the model center and the intersection $g$ of the gaze direction with a plane $P$ that passes through the model center and faces the user (with the up vector determined by the camera). Rotation is counterclockwise about an axis obtained by rotating $\vec{r}$ counterclockwise $90^{\circ}$ in $P$. For scaling, size is increased (decreased) when $g$ is above/right of (below/left of) the line $u=-v$, where $u$ and $v$ are coordinates in $P$. To perform combined translation and rotation, the virtual model is rigidly coupled to the translation and orientation of the user's head, accomplishing zero-order control.

\section{CONCLUSIONS AND FUTURE WORK}

We have described a hands-free user interface for manipulating virtual 3D content in AR, designed for intraoperative use with input based on voice and head tracking. We are currently running a user study to determine the effectiveness of our techniques.

\section{ACKNOWLEDGMENTS}

This material is based on work supported in part by the National Science Foundation under Grant IIS-1514429 (S. Feiner, PI), the National Institutes of Health under Grant NHLBI: 5T35HL007616-37 (R. Leibel, PI), and Columbia University Vagelos College of Physicians and Surgeons under Dean's Research Fellowships to G. Loeb and S. Sadri. We thank the physicians at NewYork-Presbyterian/Columbia University Medical Center who contributed to this research.

\section{REFERENCES}

A. Grinshpoon, S. Sadri, G. Loeb, C. Elvezio, and S. Feiner. 2018. Hands-free interaction for augmented reality in vascular interventions. In Proc. IEEE Virtual Reality.

M. Hasan and H. Yu. 2017. Innovative developments in HCI and future trends. Int. F. of Automat. and Comp. 14, 1 (Feb 2017), 10-20. https://doi.org/10.1007/ s11633-016-1039-6

S. Jalaliniya, J. Smith, M. Sousa, L. Büthe, and T. Pederson. 2013. Touch-less interaction with medical images using hand \& foot gestures. In Proc. UbiComp 2013 Adjunct. ACM Press, NY, NY, 1265-1274. https://doi.org/10.1145/2494091.2497332

S. M. LaValle, A. Yershova, M. Katsev, and M. Antonov. 2014. Head tracking for the Oculus Rift. In Proc. ICRA 2014. 187-194. https://doi.org/10.1109/ICRA.2014.6906608

G. Loeb, S. Sadri, A. Grinshpoon, J. Carroll, C. Cooper, C. Elvezio, S. Mutasa, G. Mandigo, S. Lavine, J. Weintraub, A. Einstein, S. Feiner, and P. Meyers. 2018. 3:54 PM Abstract No. 29 Augmented reality guidance for cerebral angiography. FVIR 29, 4 (Apr 2018), S17. https://doi.org/10.1016/j.jvir.2018.01.036

A. Mewes, B. Hensen, F. Wacker, and C. Hansen. 2017. Touchless interaction with software in interventional radiology and surgery: A systematic literature review. Int. f. Comput. Assist. Radiol. Surg. 12, 2 (Feb 2017), 291-305. https://doi.org/10. 1007/s11548-016-1480-6

A. Nishikawa, T. Hosoi, K. Koara, D. Negoro, A. Hikita, S. Asano, H. Kakutani, F. Miyazaki, M. Sekimoto, M. Yasui, Y. Miyake, S. Takiguchi, and M. Monden. 2003. FAce MOUSe: A novel human-machine interface for controlling the position of a laparoscope. IEEE Transactions on Robotics and Automation 19, 5 (Oct 2003), 825-841. https://doi.org/10.1109/TRA.2003.817093

R. Reilink, G. de Bruin, M. Franken, M. A. Mariani, S. Misra, and S. Stramigioli. 2010. Endoscopic camera control by head movements for thoracic surgery. In Proc. IEEE RAS \& EMBS Int. Conf. on Biomed. Robotics and Biocmechatronics. IEEE, 510-515. https://doi.org/10.1109/BIOROB.2010.5627043

J. P. Wachs, K. Vujjeni, E. T. Matson, and S. Adams. 2010. "A window on tissue"-Using facial orientation to control endoscopic views of tissue depth. Proc. IEEE EMBC 20102010 (2010), 935-938. https://doi.org/10.1109/iembs.2010.5627538 